\title{
Europäischer Tarifbericht des WSI - 2011/2012
}

Die Entwicklung der Löhne steht gegenwärtig in den meisten europäischen Ländern unter dem Vorzeichen anhaltender Reallohnverluste. Vor dem Hintergrund einer hohen Arbeitslosigkeit und einer sich deutlich abkühlenden Konjunktur haben sich die ökonomischen Rahmenbedingungen der Tarifpolitik fast überall in Europa deutlich verschlechtert. Während sich in Deutschland zaghaft eine Trendwende zu expansiveren Lohnzuwächsen andeutet, wird unter dem Druck der Europäischen Union (EU) insbesondere in den südeuropäischen Krisenstaaten nach wie vor eine Politik des Einfrierens oder gar der Kürzung von Löhnen propagiert. Mit der Unterordnung der Lohnpolitik unter die Wettbewerbspolitik droht eine allgemeine Absenkungsspirale, die die ökonomische Krise und Stagnation in Europa weiter verschärft.

THORSTEN SCHULTEN

\section{1. Ökonomische Rahmenbedingungen der Tarifpolitik}

\subsection{Allgemeine Wirtschaftsentwicklung}

Die konjunkturellen Rahmenbedingungen für Tarifauseinandersetzungen in Europa haben sich im Jahr 2011 insgesamt wieder verschlechtert. Nachdem in den meisten europäischen Ländern die schwere Wirtschaftskrise 2008/2009 angesichts der sich deutlich erholenden Wirtschaftskonjunktur im Jahr 2010 bereits überwunden schien, hat sich die konjunkturelle Dynamik 2011 erneut verlangsamt. Vor dem Hintergrund einer strikten Austeritätspolitik und einer sich merklich abkühlenden Weltkonjunktur wird für 2012 für mehrere europäische Länder erneut eine Rezession vorausgesagt, während für die Europäische Union (EU 27) insgesamt eine Stagnation der Wirtschaftsentwicklung prognostiziert wird (vgl. Europäische Kommission 2012; IMK et al. 2012).

Im EU-Durchschnitt stieg das Bruttoinlandsprodukt (BIP) 2011 um 1,5\%. Im Vorjahr lag das EU-weite Wirtschaftswachstum demgegenüber noch bei 2,0 \%. Für 2012 erwartet die Europäische Kommission für die gesamte EU ein Nullwachstum (Tabelle 1). ${ }^{\bullet}$ Zwischen den einzelnen EU-Staaten zeigt der Konjunkturverlauf jedoch sehr große Unterschiede. So gab es 2011 eine Reihe von Staaten mit relativ hohen Wachstumsraten. An der Spitze standen mit einem BIP-Wachstum zwischen 5,5 und 7,6 \% die baltischen Staaten, die sich nach einem starken ökonomischen Einbruch in den Krisenjahren 2008/2009 wieder deutlich erholen konnten. Ein mit 4,3\% recht robustes Wachstum gab es auch in Polen, das als einziges Land in Europa während der Weltwirtschaftskrise keinen Einbruch erlebte und sich gegen den allgemeinen Trend in einer bereits seit mehreren Jahren andauernden Aufschwungphase befindet. Darüber hinaus kam es 2011 auch in einigen nord- und westeuropäischen Ländern (Finnland und Schweden sowie Deutschland und Österreich) mit Zuwachsraten zwischen 2,9 und 3,9 \% zu einem relativ kräftigen Wachstum. Dagegen zeigten Großbritannien und Irland sowie die Mehrzahl der südeuropäischen Staaten eine relativ schwache Wirtschaftsentwicklung. Das Schlusslicht bildeten Portugal und Griechenland, deren BIP um 1,6 \% bzw. sogar 6,9 \% zurückging.

\footnotetext{
Im Folgenden wird, falls nicht anders ausgewiesen, auf Daten der Annual Macro-Economic Database (AMECO) zurückgegriffen, die von der Generaldirektion Wirtschaft und Finanzen (GD ECFIN) der Europäischen Kommission bereitgestellt wird (http://ec.europa.eu/economy_finance/db_indicators/ameco/index_en.htm). Bei den Angaben für 2011 handelt es sich teilweise noch um vorläufige Daten. Bei den Angaben für 2012 handelt es sich um Prognosedaten der Europäischen Kommission (vgl. Europäische Kommission 2012).
} 
TABELLE 1

Wachstum und Arbeitslosigkeit in der Europäischen Union 2009-2012*

\begin{tabular}{|c|c|c|c|c|c|c|c|c|}
\hline & \multicolumn{4}{|c|}{ Bruttoinlandsprodukt } & \multicolumn{4}{|c|}{ Arbeitslosigkeit } \\
\hline & 2009 & 2010 & 2011 & 2012 & 2009 & 2010 & 2011 & 2012 \\
\hline \multicolumn{9}{|l|}{ Nordeuropa } \\
\hline Dänemark & $-5,8$ & 1,3 & 1,0 & 1,1 & 6,0 & 7,5 & 7,6 & 7,7 \\
\hline Finnland & $-8,4$ & 3,7 & 2,9 & 0,8 & 8,2 & 8,4 & 7,8 & 7,9 \\
\hline Schweden & $-5,0$ & 6,1 & 3,9 & 0,3 & 8,3 & 8,4 & 7,5 & 7,7 \\
\hline \multicolumn{9}{|l|}{ Westeuropa } \\
\hline Belgien & $-2,8$ & 2,3 & 1,9 & $-0,0$ & 7,9 & 8,3 & 7,2 & 7,6 \\
\hline Deutschland & $-5,1$ & 3,7 & 3,0 & 0,7 & 7,8 & 7,1 & 5,9 & 5,5 \\
\hline Frankreich & $-2,7$ & 1,5 & 1,7 & 0,5 & 9,5 & 9,8 & 9,7 & 10,2 \\
\hline Großbritannien & $-4,4$ & 2,1 & 0,7 & 0,5 & 7,6 & 7,8 & 8,0 & 8,5 \\
\hline Irland & $-7,0$ & $-0,4$ & 0,7 & 0,5 & 11,9 & 13,7 & 14,4 & 14,3 \\
\hline Luxemburg & $-5,3$ & 2,7 & 1,6 & 1,1 & 5,1 & 4,6 & 4,8 & 5,2 \\
\hline Niederlande & $-3,5$ & 1,7 & 1,2 & $-0,9$ & 3,7 & 4,5 & 4,4 & 5,7 \\
\hline Österreich & $-3,8$ & 2,3 & 3,1 & 0,8 & 4,8 & 4,4 & 4,2 & 4,3 \\
\hline \multicolumn{9}{|l|}{ Südeuropa } \\
\hline Griechenland & $-3,3$ & $-3,5$ & $-6,9$ & $-4,7$ & 9,5 & 12,6 & 17,7 & 19,7 \\
\hline Italien & $-5,5$ & 1,8 & 0,4 & $-1,4$ & 7,8 & 8,4 & 8,4 & 9,5 \\
\hline Malta & $-2,7$ & 2,3 & 2,1 & 1,2 & 6,9 & 6,9 & 6,5 & 6,6 \\
\hline Portugal & $-2,9$ & 1,4 & $-1,6$ & $-3,3$ & 10,6 & 12,0 & 12,9 & 15,5 \\
\hline Spanien & $-3,7$ & $-0,1$ & 0,7 & $-1,8$ & 18,0 & 20,1 & 21,7 & 24,4 \\
\hline Zypern & $-1,9$ & 1,1 & 0,5 & $-0,8$ & 5,3 & 6,2 & 7,8 & 9,8 \\
\hline \multicolumn{9}{|l|}{ Osteuropa } \\
\hline Bulgarien & $-5,5$ & 0,4 & 1,7 & 0,5 & 6,8 & 10,2 & 11,2 & 12,0 \\
\hline Estland & $-14,3$ & 2,3 & 7,6 & 1,6 & 13,8 & 16,9 & 12,5 & 11,6 \\
\hline Lettland & $-17,7$ & $-0,3$ & 5,5 & 2,2 & 17,1 & 18,7 & 16,1 & 14,8 \\
\hline Litauen & $-14,8$ & 1,4 & 5,9 & 2,4 & 13,7 & 17,8 & 15,4 & 13,8 \\
\hline Polen & 1,6 & 3,9 & 4,3 & 2,7 & 8,2 & 9,6 & 9,7 & 9,8 \\
\hline Rumänien & $-6,6$ & $-1,6$ & 2,5 & 1,4 & 6,9 & 7,3 & 7,4 & 7,2 \\
\hline Slowakei & $-4,9$ & 4,2 & 3,3 & 1,8 & 12,0 & 14,4 & 13,5 & 13,2 \\
\hline Slowenien & $-8,0$ & 1,4 & $-0,2$ & $-1,4$ & 5,9 & 7,3 & 8,2 & 9,1 \\
\hline Ungarn & $-6,8$ & 1,3 & 1,7 & $-0,3$ & 10,0 & 11,2 & 10,9 & 10,6 \\
\hline Tschechien & $-4,7$ & 2,7 & 1,7 & 0,0 & 6,7 & 7,3 & 6,7 & 7,2 \\
\hline Gesamte EU 27 & $-4,3$ & 2,0 & 1,5 & 0,0 & 9,0 & 9,7 & 9,7 & 10,3 \\
\hline
\end{tabular}

*Bruttoinlandsprodukt = Veränderung des BIP in \% gegenüber dem Vorjahr.

Arbeitslosenquote = Anzahl der Arbeitslosen in \% der zivilen Erwerbsbevölkerung (Eurostat-Definition).

Angaben für 2012: Prognose der Europäischen Kommission.

Quelle: AMECO Datenbank der Europäischen Komission, Version: 11.5.2012.

Für 2012 prognostiziert die Europäische Kommission für fast alle EU-Staaten einen deutlichen Wachstumseinbruch. In acht Ländern wird dabei sogar mit einem Wachstumsrückgang gerechnet. Darunter befinden sich neben den Niederlanden, Slowenien und Ungarn vor allem die südeuropäischen Länder, wobei wiederum für Portugal und Griechenland mit 3,3 bzw. 4,7 \% die stärksten Wachstumsrückgänge erwartet werden. Die sich weiter verschärfende ökonomische Krise in Südeuropa scheint 2012 auch deutliche Rückwirkungen auf die Konjunkturentwicklung in den exportorientierten Ländern Nord- und Westeuropas zu entfalten, wo mit Ausnahme von Dänemark und Luxemburg die prognostizierten BIP-Zuwächse überall zumeist deutlich unterhalb der Ein-Prozent-Marke bleiben. Auch Deutschland, das sich in den letzten beiden Jahren mit hohen Wachstumsraten gut von der Krise 2009 erholt hatte, wird 2012 mit prognostizierten 0,7 \% BIP-Zuwachs von einem deutlichen Wachstumseinbruch betroffen sein und gehört damit in Europa nicht länger zur „Insel der Glückseeligen“" (Deutsche Bank Research 2011).

\subsection{Die Lage auf dem Arbeitsmarkt}

Neben der allgemeinen konjunkturellen Entwicklung ist die Lage auf dem Arbeitsmarkt ein weiterer zentraler Faktor für die Tarifauseinandersetzungen, der sich direkt auf die Verhandlungs- und Durchsetzungsmacht der Tarifvertragsparteien auswirkt. In vielen europäischen Ländern wird derzeit die Position der Gewerkschaften durch eine anhaltend hohe Arbeitslosigkeit geschwächt. Im Jahresdurchschnitt 2011 waren EU-weit mehr als 23 Mio. Menschen offiziell arbeitslos, was einer Arbeitslosenquote von 9,7\% entspricht (Tabelle 1). Für 2012 wird angesichts der rückläufigen Konjunkturprognosen ein weiterer Anstieg der Arbeitslosigkeit erwartet. So lag die Arbeitslosenquote im Mai 2012 im EU-Durchschnitt bereits bei 10,3 \%, womit fast 25 Mio. Menschen innerhalb der EU offiziell als arbeitslos registriert wurden (Eurostat 2012).

Spiegelbildlich zur Konjunkturlage bestehen auch bei den Arbeitslosenquoten zwischen den einzelnen EU-Staaten sehr große Unterschiede. Eine extrem hohe Arbeitslosigkeit mit Quoten von zum Teil deutlich über $10 \%$ existiert neben Irland vor allem in einer Reihe süd- und osteuropäischer Länder. Spitzenreiter sind hierbei Griechenland und Spanien, die mit 17,7 bzw. 21,7 \% bereits 2011 die höchsten Werte aufwiesen. Ein nochmaliger kräftiger Anstieg der Arbeitslosenquoten wird in diesen Ländern für 2012 prognostiziert. Am anderen Ende derSkalabefindet sich eine Reihe westeuropäischer Länder, die über eine vergleichsweise günstige Situation auf dem Arbeitsmarkt verfügen. Mit Luxemburg, den Niederlanden und Österreich lag die Arbeitslosenquote im Jahr 2011 in drei kleineren westeuropäischen Ländern noch unter $5 \%$. Mit der offenen Rezession in den Niederlanden wird jedoch auch dort 2012 ein spürbarer Anstieg der Arbeitslosigkeit erwartet. Gegen den Trend vollzieht sich bislang die Entwicklung in Deutschland, wo die Arbeitslosenquote seit 2009 kontinuierlich zurückgegangen ist und auch für 2012 eine Fortsetzung dieser Entwicklung prognostiziert wird.

\subsection{Preis- und Produktivitätsentwicklung}

Die Tarifauseinandersetzungen in Europa orientieren sich traditionell neben der Konjunkturentwicklung und der Lage auf dem Arbeitsmarkt vor allem an der Entwicklung der Preise und der Arbeitsproduktivität (Tabelle 2). Die Summe beider Indikatoren bildet den lohnpolitischen Verteilungsspielraum, dessen Ausschöpfung durch entsprechende Lohn- 
Preise, Arbeitsproduktivität und Verteilungsspielraum in der Europäischen Union 2009-2012*

\begin{tabular}{|c|c|c|c|c|c|c|c|c|c|c|c|c|}
\hline & \multicolumn{4}{|c|}{ Preise } & \multicolumn{4}{|c|}{ Arbeitsproduktivität } & \multicolumn{4}{|c|}{ Verteilungsspielraum } \\
\hline & 2009 & 2010 & 2011 & 2012 & 2009 & 2010 & 2011 & 2012 & 2009 & 2010 & 2011 & 2012 \\
\hline \multicolumn{13}{|l|}{ Nordeuropa } \\
\hline Dänemark & 1,1 & 2,2 & 2,7 & 2,6 & $-2,7$ & 3,6 & 1,5 & 1,0 & $-1,6$ & 5,8 & 4,1 & 3,6 \\
\hline Finnland & 1,6 & 1,7 & 3,3 & 3,0 & $-5,9$ & 4,9 & 1,7 & 0,9 & $-4,2$ & 6,6 & 5,0 & 3,9 \\
\hline Schweden & 1,9 & 1,9 & 1,4 & 1,1 & $-2,7$ & 5,0 & 1,7 & 0,1 & $-0,7$ & 6,9 & 3,1 & 1,3 \\
\hline \multicolumn{13}{|l|}{ Westeuropa } \\
\hline Belgien & 0,0 & 2,3 & 3,5 & 2,9 & $-2,7$ & 1,4 & 0,5 & $-0,1$ & $-2,7$ & 3,8 & 4,0 & 2,8 \\
\hline Deutschland & 0,2 & 1,2 & 2,5 & 2,3 & $-5,2$ & 3,2 & 1,6 & $-0,4$ & $-4,9$ & 4,4 & 4,1 & 2,0 \\
\hline Frankreich & 0,1 & 1,7 & 2,3 & 2,1 & $-1,3$ & 1,4 & 1,0 & 0,0 & $-1,2$ & 3,2 & 3,3 & 2,1 \\
\hline Großbritannien & 2,2 & 3,3 & 4,5 & 2,9 & $-2,8$ & 1,9 & 0,2 & 0,1 & $-0,7$ & 5,2 & 4,7 & 3,0 \\
\hline Irland & $-1,7$ & $-1,6$ & 1,2 & 1,7 & 1,2 & 4,0 & 2,8 & 1,2 & $-0,5$ & 2,4 & 4,0 & 2,9 \\
\hline Luxemburg & 0,0 & 2,8 & 3,7 & 3,0 & $-6,2$ & 0,8 & $-1,1$ & $-0,9$ & $-6,2$ & 3,6 & 2,6 & 2,1 \\
\hline Niederlande & 1,0 & 0,9 & 2,5 & 2,5 & $-2,5$ & 2,2 & 1,0 & $-0,7$ & $-1,5$ & 3,1 & 3,4 & 1,8 \\
\hline Österreich & 0,4 & 1,7 & 3,6 & 2,4 & $-2,5$ & 1,7 & 1,6 & 0,3 & $-2,1$ & 3,4 & 5,2 & 2,8 \\
\hline \multicolumn{13}{|l|}{ Südeuropa } \\
\hline Griechenland & 1,3 & 4,7 & 3,1 & $-0,5$ & $-3,0$ & $-1,7$ & $-0,2$ & 0,1 & $-1,7$ & 3,0 & 2,9 & $-0,5$ \\
\hline Italien & 0,8 & 1,6 & 2,9 & 3,2 & $-2,7$ & 2,7 & 0,3 & $-0,4$ & $-2,0$ & 4,4 & 3,2 & 2,9 \\
\hline Malta & 1,8 & 2,0 & 2,4 & 2,0 & $-2,4$ & 0,0 & $-0,3$ & 0,6 & $-0,6$ & 2,0 & 2,1 & 2,7 \\
\hline Portugal & $-0,9$ & 1,4 & 3,6 & 3,0 & $-0,3$ & 3,0 & $-0,1$ & 0,0 & $-1,2$ & 4,4 & 3,5 & 3,1 \\
\hline Spanien & $-0,2$ & 2,0 & 3,1 & 1,9 & 2,9 & 2,6 & 2,8 & 2,0 & 2,7 & 4,7 & 5,9 & 3,9 \\
\hline Zypern & 0,2 & 2,6 & 3,5 & 3,4 & $-1,3$ & 1,1 & $-0,1$ & 0,7 & $-1,1$ & 3,7 & 3,4 & 4,1 \\
\hline \multicolumn{13}{|l|}{ Osteuropa } \\
\hline Bulgarien & 2,5 & 3,0 & 3,4 & 2,6 & $-2,9$ & 5,3 & 6,1 & 2,4 & $-0,5$ & 8,4 & 9,5 & 5,0 \\
\hline Estland & 0,2 & 2,7 & 5,1 & 3,9 & $-4,7$ & 7,4 & 0,6 & 1,0 & $-4,5$ & 10,1 & 5,7 & 4,9 \\
\hline Lettland & 3,3 & $-1,2$ & 4,2 & 2,6 & $-5,3$ & 4,7 & 2,0 & 1,5 & $-2,0$ & 3,5 & 6,3 & 4,1 \\
\hline Litauen & 4,2 & 1,2 & 4,1 & 3,1 & $-8,6$ & 6,9 & 3,8 & 1,8 & $-4,4$ & 8,1 & 7,9 & 4,9 \\
\hline Polen & 4,0 & 2,7 & 3,9 & 3,7 & 1,2 & 3,4 & 3,3 & 2,4 & 5,2 & 6,1 & 7,2 & 6,1 \\
\hline Rumänien & 5,6 & 6,1 & 5,8 & 3,1 & $-4,7$ & $-0,2$ & 2,0 & 0,9 & 0,9 & 5,9 & 7,9 & 4,0 \\
\hline Slowakei & 0,9 & 0,7 & 4,1 & 2,9 & $-3,0$ & 5,8 & 1,5 & 1,4 & $-2,1$ & 6,5 & 5,6 & 4,3 \\
\hline Slowenien & 0,9 & 2,1 & 2,1 & 2,2 & $-6,3$ & 4,0 & 1,6 & 0,3 & $-5,4$ & 6,1 & 3,6 & 2,5 \\
\hline Tschechien & 0,6 & 1,2 & 2,1 & 3,3 & $-3,5$ & 4,5 & 1,4 & 0,1 & $-2,9$ & 5,7 & 3,5 & 3,4 \\
\hline Ungarn & 4,0 & 4,7 & 3,9 & 5,5 & $-4,2$ & 0,9 & 1,4 & $-1,3$ & $-0,1$ & 5,7 & 5,3 & 4,3 \\
\hline Gesamte EU 27 & 1,0 & 2,1 & 3,1 & 2,6 & $-2,4$ & 2,5 & 1,4 & 0,4 & $-1,4$ & 4,6 & 4,5 & 2,9 \\
\hline
\end{tabular}

${ }^{*}$ Preise $=$ Harmonisierter Verbraucherpreisindex (HVPI), Veränderung in \% gegenüber dem Vorjahr.

Arbeitsproduktivität = Reales BIP pro Erwerbstätiger, Veränderung in \% gegenüber dem Vorjahr.

Verteilungsspielraum = Summe aus Preis- und Arbeitsproduktivitätsentwicklung.

Angaben für 2012: Prognose der Europäischen Kommission.

Quelle: AMECO Datenbank der Europäischen Kommission, Version: 11.5.2012; Berechnungen des WSI.

erhöhungen den Beschäftigten eine gleichgewichtige Partizipation an der allgemeinen Wirtschaftsentwicklung garantiert und das Verhältnis zwischen Arbeits- und Kapitaleinkommen konstant hält. Bei der Definition des lohnpolitischen Verteilungsspielraumes als Summe aus Preis- und Produktivitätsentwicklung ist in der Wirtschaftswissenschaft strittig, welche konkreten Indikatoren insbesondere für die Preisentwicklung verwendet werden sollen. Gegenüber möglichen alternativen Indikatoren (wie z. B. der Kerninflationsrate, der Zielinflationsrate der Europäischen Zentralbank
(EZB) oder der Entwicklung der Erzeugerpreise) wird im Folgenden bei der Bestimmung der nationalen Verteilungsspielräume die jeweils nationale Entwicklung der Verbraucherpreise zugrunde gelegt, wobei für den internationalen Vergleich der Harmonisierte Verbraucherpreisindex (HVPI) als Grundlage verwendet wird. Aus Arbeitnehmersicht stellen die Verbraucherpreise den wichtigsten ökonomischen Indikator dar, weil sie über das reale Lohnniveau und die damit verbundene Kaufkraft entscheiden. Dementsprechend bilden die nationalen Verbraucherpreise für die Ge- 
werkschaften die bedeutsamste Orientierungsgröße bei den Tarifauseinandersetzungen.

2011 kam es trotz einer sich abschwächenden Konjunkturentwicklung zu einem relativ starken Anstieg der Verbraucherpreise, der vor allem auf den Anstieg der Energiepreise zurückzuführen ist. Im EU-Durchschnitt lag die Inflationsrate bei 3,1\% und damit einen ganzen Prozentpunkt höher als im Vorjahr (Tabelle 2). Den höchsten Preisauftrieb gab es mit 5,8 \% in Rumänien, gefolgt von 5,1 \% in Estland und 4,5 \% in Großbritannien. Trotz Rezession kam es auch in Griechenland und Portugal mit Werten von 3,1 bzw. 3,6 \% zu ungewöhnlich hohen Preissteigerungen. Die niedrigsten Inflationsraten gab es demgegenüber mit 1,2 bzw. 1,4 \% in Irland und Schweden. Für 2012 erwartet die Europäische Kommission trotz EU-weiter Stagnation einen Anstieg der Verbraucherpreise von durchschnittlich 2,6 \%.

Die Entwicklung der Arbeitsproduktivität hat $2011 \mathrm{mit}$ einem Zuwachs von EU-weit lediglich 1,4 \% wieder deutlich an Dynamik verloren und liegt um mehr als einen Prozentpunkt unterhalb des Wertes des Vorjahres (Tabelle 2). Die höchsten Produktivitätszuwächse konnten einige osteuropäische Staaten verzeichnen, darunter Polen und Litauen mit Werten von 3,3 bzw. 3,8 \% sowie Bulgarien, das als einsamer Spitzenreiter einen Anstieg von 6,1 \% verzeichnete. Während in der Mehrzahl der EU-Staaten die Produktivitätszuwächse zwischen 1-2 \% schwankten, waren sie in Griechenland und Portugal leicht und in Luxemburg signifikant negativ. Nach den Vorhersagen der Europäischen Kommission wird das Produktivitätswachstum 2012 deutlich zurückgehen und im EU-Durchschnitt nur noch bei 0,4 \% liegen.

Da sich der Anstieg der Verbraucherpreise und der Rückgang der Arbeitsproduktivität im Jahr 2011 ungefähr die Waage hielten, hat sich rechnerisch der lohnpolitische Verteilungsspielraum im EU-Durchschnitt mit 4,5\% gegenüber 4,6 \% im Vorjahr kaum verändert (Tabelle 2). Der größte Verteilungsspielraum existierte mit 9,5\% in Bulgarien, gefolgt von einer Reihe weiterer osteuropäischer Länder mit Werten zwischen 5 und $8 \%$. Diese Länder haben damit die ökonomischen Voraussetzungen geschaffen, den lohnpolitischen Aufholprozess gegenüber Westeuropa wieder aufzunehmen, der zuvor durch die Krise unterbrochen bzw. teilweise sogar rückgängig gemacht worden war. Lediglich in Slowenien und Tschechien lag der Verteilungsspielraum mit Werten von 3,6 bzw. 3,5 \% unterhalb des EU-Durchschnittes. Das gleiche gilt auch für die Mehrheit der Staaten aus Nord-, West- und Südeuropa. Außerhalb Osteuropas verfügten lediglich Finnland, Großbritannien, Österreich und Spanien mit Werten zwischen 4,7 und 5,9\% über einen Verteilungsspielraum oberhalb des EU-Durchschnittes.

Angesichts einer rückläufigen Preisentwicklung und eines starken Rückgangs der Produktivitätszuwächse wird sich der lohnpolitische Verteilungsspielraum 2012 deutlich verringern. Aus den Prognosen der Europäischen Kommission ergibt sich im EU-Durchschnitt ein Wert von 2,9\%, wobei dieser vor allem durch die niedrigen Verteilungsspielräume in den westeuropäischen Ländern nach unten ge- drückt wird. In den meisten osteuropäischen Ländern sowie in einigen nord- und südeuropäischen Staaten liegt der Verteilungsspielraum hingegen nach wie vor zum Teil deutlich oberhalb von $3 \%$.

Während hier für die Berechnung des jeweils jahresbezogenen Verteilungspielraumes auch die jahresbezogene Produktivität verwendet wird, wird für eine gesamtwirtschaftlich ausgerichtete Tarifpolitik in der Regel eine Orientierung an der Trendproduktivität empfohlen, die sich aus der durchschnittlichen Produktivitätsentwicklung mehrerer Jahre ergibt (vgl. z. B. Herr/Horn 2012). Eine solche mittelfristige Orientierung hat den Vorteil, dass sie zu einer Verstetigung der Lohnpolitik beitragen kann und prozyklische Effekte vermeidet, die sich z. B. dann ergeben können, wenn eine rückläufige Konjunkturentwicklung durch geringe Lohnzuwächse oder gar Lohnkürzungen weiter verstärkt wird. Legt man z. B. die durchschnittliche Produktivitätsentwicklung der letzten zehn Jahre zugrunde, die bei etwa 1,4 \% liegt, so ergibt sich für die Jahre 2011 und 2012 im EU-Durchschnitt ein Verteilungsspielraum von 4,0 bis 4,5 \%. Während dieser aufgrund der nachholenden Entwicklung in den meisten osteuropäischen Ländern deutlich größer ausfällt, liegt er im übrigen Europa zumeist zwischen 3 und $4 \%$.

\section{Entwicklung der Tariflöhne}

\subsection{Der EZB-Tariflohnindikator}

Die Analyse der Tariflohnentwicklung in Europa steht vor dem grundsätzlichen Problem, dass es bis heute keine offizielle europäische Tariflohnstatistik gibt. ${ }^{2}$ Die einzige offizielle Datenquelle auf europäischer Ebene bildet bislang der sogenannte „Indikator der Tarifverdienste“, der von der Europäischen Zentralbank (EZB) als aggregierte Größe für die gesamte Euro-Zone berechnet wird. Demnach sind die Tariflöhne im Euroraum im Jahr 2011 nominal durch-

(2) Seit einigen Jahren veröffentlicht das European Industrial Relations Observatory (EIRO) regelmäßig Daten über die Entwicklung der „Tariflöhne" in Europa. Nachdem die Berichte lange Zeit große methodische Mängel aufwiesen, die u.a. darin lagen, dass nicht immer klar zwischen Tarif- und Effektivlohndaten unterschieden worden ist, wurde der Bericht im Jahr 2011 erstmals auf eine neue verbesserte Datengrundlage gestellt (Aumayr et al. 2011). Hierbei wird ähnlich wie in dem vorliegenden Bericht auf die wenigen verfügbaren nationalen Datenquellen überTariflöhne zurückgegriffen. Qualitative Berichte über dieTarifauseinandersetzungen in den einzelnen EU-Staaten finden sich in den laufenden nationalen Beiträgen zum EIRO (http://www. eurofound.europa.eu/eiro) sowie in dem gemeinsam vom Europäischen Gewerkschaftsinstitut und dem Amsterdam Institute for Advanced Labour Studies (AIAS) herausgegebenen Collective Bargaining Newsletter (http://www.etui. org/E-Newsletters/Collective-bargaining-newsletter). 
schnittlich um $2 \%$ angestiegen (Abbildung 1). Die EZBDaten für das 1. Quartal 2012 deuten darauf hin, dass sich die Tariflohnentwicklung auch 2012 in ähnlichem Tempo fortsetzen wird. Nach Abzug der Preissteigerungsrate gehen die Tariflöhne real jedoch in beiden Jahren um 0,7 \% zurück.

Über die gesamte letzte Dekade hinweg zeigt die Entwicklung des EZB-Tariflohnindikators sowohl nominal als auch real eine erstaunliche Konstanz. Während die nominale Tariflohnentwicklung zwischen 2,1 und 2,7 \% schwankt, liegt sie real zumeist wenige Zehntel-Prozent oberhalb der Inflationsrate. Eine Ausnahme bildeten lediglich das Jahr 2008, wo es vor dem Hintergrund steigender Preise auch zu einem ungewöhnlich starken nominalen Tariflohnanstieg kam, sowie das Krisenjahr 2009, in dem der Preisanstieg extrem niedrig war und damit erstmals zu einem kräftigen Anstieg der realen Tarifverdienste führte.

Bei dem EZB-Tariflohnindikator handelt es sich um sogenannte „experimentelle Daten“, die durch die Zusammenführung nicht harmonisierter nationaler Datenquellen berechnet werden und deshalb lediglich als Näherungswert an die Wirklichkeit angesehen werden können (Schubert 2012). Bei der Berechnung des EZB-Indikators werden Tariflohndaten aus zehn Ländern berücksichtigt, die insgesamt $98 \%$ der Euro-Zone abdecken. ${ }^{3}$ Allerdings werden von der EZB weder die verwendeten nationalen Daten veröffentlicht, noch werden die zugrunde liegenden nationalen Datenquellen öffentlich preisgegeben, sodass der EZB-Tariflohnindikator insgesamt sehr intransparent und kaum nachprüfbar ist.

\subsection{Ausgewählte EU-Staaten}

Mangels einer originär europäischen Datenquelle muss bei der Analyse der Tariflohnentwicklung in Europa notgedrungen auf nicht harmonisierte nationale Datensätze zurückgegriffen werden, die in den meisten Fällen entweder von den nationalen Statistikämtern oder von den nationalen Arbeitsministerien erhoben werden und nur für eine begrenzte Anzahl von Ländern verfügbar sind. ${ }^{\circledR}$ Die Analyse der zehn hier berücksichtigten EU-Staaten zeigt insgesamt ein recht uneinheitliches Bild, was darauf hindeutet, dass die Tarifpolitik nach wie vor in erster Linie von den besonderen nationalen Rahmenbedingungen geprägt wird. Im Jahr 2011 bewegten sich in acht Ländern die nominalen Tariflohnzuwächse zwischen 1,8 und 2,6 \%. Deutschland befand sich mit einer Tariflohnsteigerung von $2 \%$ im unteren Mittelfeld. Lediglich Portugal und die Niederlande blieben mit jeweils 1,2 \% deutlich hinter dem allgemeinen Trend zurück (Abbildung 2). In sechs von zehn Staaten waren die Tariflohnzuwächse 2011 höher als im Jahr 2010, das in vielen Ländern noch durch die Tarifabschlüsse des Krisenjahres 2009 geprägt wurde (Schulten 2011a). Für 2012 deuten sich für vier Länder, darunter Belgien, Deutschland, Finnland und Österreich, noch einmal deutlich höhere Tariflohnsteigerungen zwischen 2,6 und $2,9 \%$ an, während insbesondere in den südeuropäischen
ABB. 1

\section{Entwicklung des EZB-Indikators der Tarifverdienste für die Euro-Zone 2000-2012*}

Veränderungen in Prozent zum Vorjahr

Nominal

Real

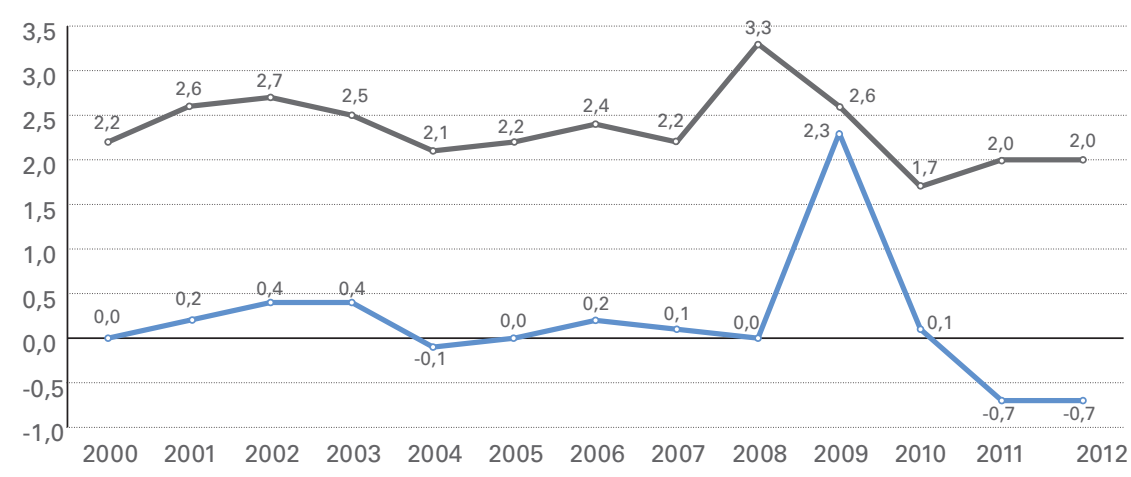

*reale Entwicklung deflationiert um den Harmonisierten Verbraucherindex (HVPI), Daten für 2012 beziehen sich auf das 1. Quartal.

Quelle: Europäische Zentralbank (http://sdw.ecb.europa.eu/browse.do?node=2120788); Berechnungen des WSI.

Krisenstaaten sowie in Frankreich die Zuwächse wesentlich niedriger ausfallen werden.

Die vergleichsweise hohen Inflationsraten haben im Jahr 2011 in neun von zehn Staaten dafür gesorgt, dass die Tariflohnbeschäftigten Reallohnverluste verkraften mussten. Einzig in Schweden kam es 2011 zu einer positiven Reallohnentwicklung bei den Tariflöhnen (Abbildung 3). 2012 könnte sich der negative Reallohntrend in mindestens sechs von zehn Staaten weiter fortsetzen. Neben Schweden dürfte es lediglich in Deutschland und Österreich eine positive Reallohnbilanz bei den Tariflöhnen geben.

Im Gegensatz zu den Tariflöhnen, die auf tarifvertraglichen Vereinbarungen beruhen, handelt es sich bei den Effektivlöhnen um diejenigen Löhne, die unabhängig von

(3) Bei den Ländern, die in dem EZB-Tariflohnindikator berücksichtigt werden, handelt es sich um Belgien, Deutschland, Finnland, Frankreich, Italien, die Niederlande, Österreich, Portugal, Slowenien und Spanien (Schubert 2012). Für Deutschland werden hierbei dieTariflohndaten der Deutschen Bundesbank benutzt, die gegenüber den im Folgenden in diesem Bericht verwendeten Daten des WSI-Tarifarchivs die Tariflohndynamik etwas unterzeichnen.

(4) Zu den nationalen Datenquellen vgl. Schulten 2011a. Im Rahmen eines von der Europäischen Kommission geförderten internationalen Forschungsprojektes über "Collectively Agreed Wages in Europe" (CAWIE), an dem das WSI als deutscher Partner und Ko-Koordinator beteiligt ist, werden derzeit die nationalen Datenquellen überTariflöhne im Hinblick auf ihre Vergleichbarkeit und Qualität analysiert und Möglichkeiten zum Aufbau einer europäischen Tariflohndatenbank sondiert. Für mehr Informationen über das CAWIEProjekt vgl. http://hiva.kuleuven.be/nl/extra/CAWIE.php. 
ABB. 2

\section{Nominale Entwicklung der Tariflöhne 2010-2012*}

Veränderungen in Prozent zum Vorjahr

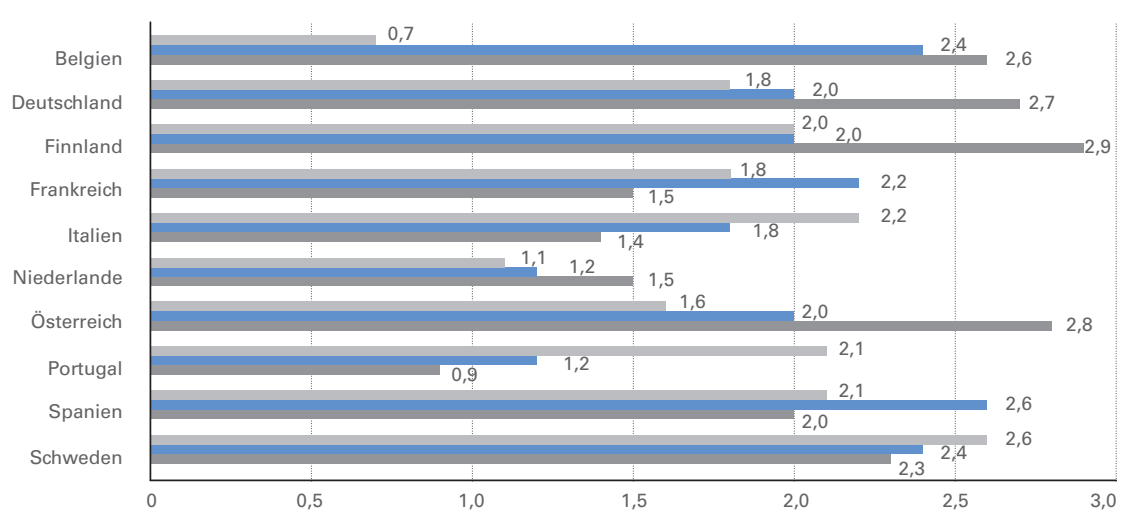

*2012: vorläufige Daten auf der Grundlage des ersten bzw. der ersten beiden Quartale. Quelle: Nationale Angaben, Berechnungen des WSI.

der jeweiligen Vertragsform tatsächlich an die Beschäftigten gezahlt wurden. In der Praxis können oft erhebliche Unterschiede zwischen der durchschnittlichen Tarif- und der durchschnittlichen Effektivlohnentwicklung bestehen. Diese als Lohndrift bezeichneten Unterschiede können verschiede-

ABB. 3

Reale Entwicklung der Tariflöhne 2010-2012*

Veränderungen in Prozent zum Vorjahr

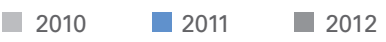

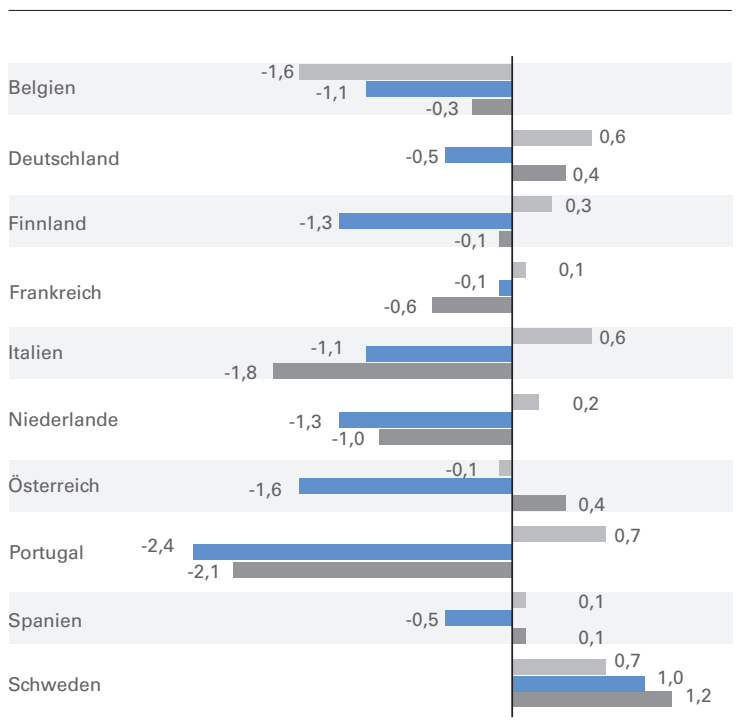

*deflationiert um den Harmonisierten Verbraucherpreisindex (HVPI). 2012: vorläufige Daten auf der Grundlage des 1. bzw. der ersten beiden Quartale; HVPI: Prognosedaten der Europäischen Kommission.

Quelle: Nationale Angaben, Berechnungen des WSI. ne Ursachen haben. Ein wichtiger Faktor hierbei ist die Tarifbindung der Beschäftigten, die innerhalb Europas eine große Spannbreite zwischen mehr als $90 \%$ in einigen nord-, west- und südeuropäischen Ländern bis hin zu weniger als $20 \%$ in einigen osteuropäischen Staaten aufweist (Schulten 2010). Darüber hinaus besteht in einigen Ländern wie z. B. in Deutschland die Möglichkeit, unter bestimmten Bedingungen auf betrieblicher Ebene von sektoralen Tariferhöhungen nach unten hin abzuweichen (Keune 2011). Umgekehrt werden in vielen Ländern von den Unternehmen nach wie vor übertarifliche Leistungen bezahlt, die zu einer positiven Lohndrift führen können. Schließlich wird das Verhältnis von Tarif- und Effektivlöhnen auch durch die Entwicklung der effektiven Arbeitszeit beeinflusst, wobei z. B. Überstunden eine positive und Kurzarbeit hingegen eine negative Lohndrift befördern.

Der Vergleich der Tariflohnzuwächse mit den in der volkswirtschaftlichen Gesamtrechnung ausgewiesenen Erhöhungen der Effektivlöhne kommt zu dem Ergebnis, dass die Lohndrift in acht von zehn hier untersuchten Ländern in den letzten Jahren zumeist positiv ausgefallen ist (Abbildung 4). Besonders hoch fiel die Lohndrift 2011 in Belgien, Deutschland und Österreich aus, wo die Effektivlöhne um einen ganzen Prozentpunkt höher als die Tariflöhne anstiegen. Dagegen war lediglich in Portugal und Spanien die Lohndrift in den letzten Jahren durchweg stark negativ, was zum einen mit den Lohnkürzungen im öffentlichen Dienst und zum andern mit dem politischen Umbau der dortigen Flächentarifvertragssysteme zusammenhängen dürfte, die

ABB. 4

\section{Lohndrift 2010-2012*}

Angaben in Prozentpunkten
2010
2011
2012

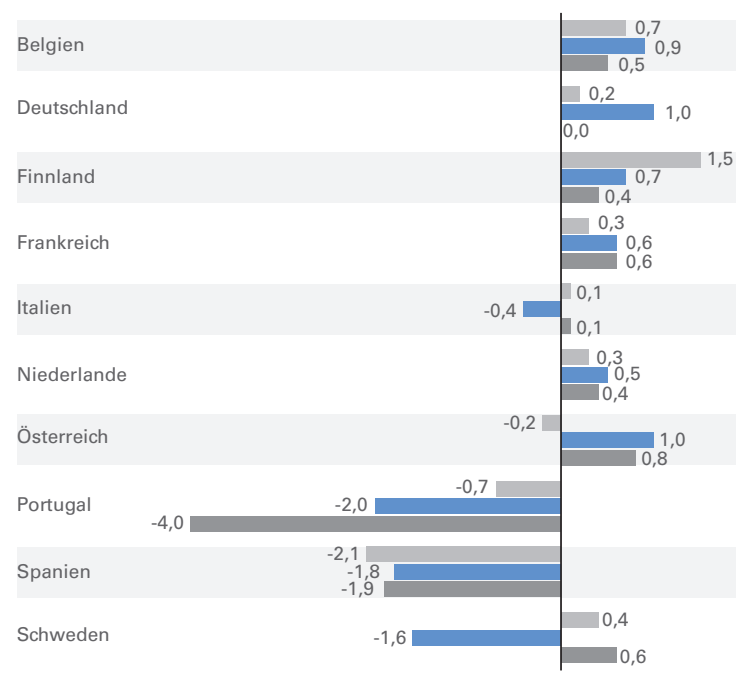

*Saldo zwischen der jährlichen nominalen Effektiv- und Tariflohnentwicklung für 2012 vorläufige und Prognosedaten. 
Lohnentwicklung und Verteilungsbilanz in der Europäischen Union 2009-2012*

\begin{tabular}{|c|c|c|c|c|c|c|c|c|c|c|c|c|}
\hline & \multicolumn{4}{|c|}{ Nominallöhne } & \multicolumn{4}{|c|}{ Reallöhne } & \multicolumn{4}{|c|}{ Verteilungsbilanz } \\
\hline & 2009 & 2010 & 2011 & 2012 & 2009 & 2010 & 2011 & 2012 & 2009 & 2010 & 2011 & 2012 \\
\hline \multicolumn{13}{|l|}{ Nordeuropa } \\
\hline Dänemark & 2,8 & 2,5 & 1,7 & 1,7 & 1,8 & 0,3 & $-0,9$ & $-0,9$ & 4,4 & $-3,2$ & $-2,4$ & $-1,9$ \\
\hline Finnland & 2,3 & 3,5 & 2,7 & 3,3 & 0,7 & 1,8 & $-0,6$ & 0,2 & 6,5 & $-3,1$ & $-2,3$ & $-0,7$ \\
\hline Schweden & 1,6 & 3,0 & 0,8 & 2,9 & $-0,3$ & 1,1 & $-0,6$ & 1,7 & 2,4 & $-3,9$ & $-2,3$ & 1,6 \\
\hline \multicolumn{13}{|l|}{ Westeuropa } \\
\hline Belgien & 1,1 & 1,4 & 3,3 & 3,1 & 1,1 & $-0,9$ & $-0,2$ & 0,3 & 3,8 & $-2,4$ & $-0,8$ & 0,3 \\
\hline Deutschland & 0,0 & 2,0 & 3,0 & 2,7 & $-0,2$ & 0,9 & 0,5 & 0,3 & 4,9 & $-2,3$ & $-1,1$ & 0,7 \\
\hline Frankreich & 1,8 & 2,1 & 2,8 & 2,1 & 1,7 & 0,4 & 0,5 & 0,0 & 3,0 & $-1,0$ & $-0,5$ & 0,0 \\
\hline Großbritannien & 2,7 & 3,6 & 1,9 & 2,4 & 0,5 & 0,3 & $-2,6$ & $-0,5$ & 3,3 & $-1,6$ & $-2,8$ & $-0,6$ \\
\hline Irland & $-1,2$ & $-3,2$ & $-1,4$ & $-0,8$ & 0,5 & $-1,6$ & $-2,6$ & $-2,5$ & $-0,7$ & $-5,6$ & $-5,4$ & $-3,6$ \\
\hline Luxemburg & 1,8 & 2,6 & 2,0 & 3,2 & 1,8 & $-0,2$ & $-1,7$ & 0,2 & 8,0 & $-1,1$ & $-0,6$ & 1,1 \\
\hline Niederlande & 2,5 & 1,4 & 1,7 & 1,9 & 1,6 & 0,4 & $-0,8$ & $-0,6$ & 4,1 & $-1,8$ & $-1,7$ & 0,1 \\
\hline Österreich & 2,2 & 1,4 & 3,0 & 3,6 & 1,8 & $-0,3$ & $-0,6$ & 1,2 & 4,3 & $-2,0$ & $-2,2$ & 0,8 \\
\hline \multicolumn{13}{|l|}{ Südeuropa } \\
\hline Griechenland & 4,0 & $-3,3$ & $-3,2$ & $-8,0$ & 2,6 & $-8,0$ & $-6,3$ & $-7,5$ & 5,6 & $-6,4$ & $-6,1$ & $-7,5$ \\
\hline Italien & 1,7 & 2,3 & 1,4 & 1,5 & 0,9 & 0,6 & $-1,5$ & $-1,7$ & 3,7 & $-2,1$ & $-1,9$ & $-1,3$ \\
\hline Malta & 3,3 & $-0,4$ & 0,4 & 1,5 & 1,5 & $-2,4$ & $-2,0$ & $-0,6$ & 3,9 & $-2,4$ & $-1,7$ & $-1,2$ \\
\hline Portugal & 2,8 & 1,4 & $-0,8$ & $-3,1$ & 3,7 & 0,0 & $-4,4$ & $-6,1$ & 4,0 & $-3,0$ & $-4,3$ & $-6,2$ \\
\hline Spanien & 4,3 & 0,0 & 0,8 & 0,1 & 4,5 & $-2,0$ & $-2,2$ & $-1,7$ & 1,6 & $-4,7$ & $-5,0$ & $-3,7$ \\
\hline Zypern & 5,3 & $-0,2$ & 2,0 & $-0,8$ & 5,1 & $-2,8$ & $-1,5$ & $-4,1$ & 6,5 & $-3,9$ & $-1,5$ & $-4,9$ \\
\hline \multicolumn{13}{|l|}{ Osteuropa } \\
\hline Bulgarien & 9,4 & 11,2 & 7,3 & 5,2 & 7,0 & 8,2 & 3,9 & 2,7 & 9,9 & 2,8 & $-2,2$ & 0,2 \\
\hline Estland & $-3,4$ & 1,4 & 1,4 & 4,0 & $-3,6$ & $-1,3$ & $-3,7$ & 0,1 & 1,2 & $-8,7$ & $-4,3$ & $-0,9$ \\
\hline Lettland & $-12,7$ & $-5,5$ & 4,2 & 1,7 & $-16,0$ & $-4,3$ & 0,0 & $-0,9$ & $-10,7$ & $-9,0$ & $-2,1$ & $-2,4$ \\
\hline Litauen & $-9,9$ & $-0,9$ & 3,4 & 2,1 & $-14,0$ & $-2,1$ & $-0,7$ & $-1,0$ & $-5,4$ & $-9,0$ & $-4,5$ & $-2,8$ \\
\hline Polen & 3,5 & 5,7 & 5,1 & 4,7 & $-0,5$ & 3,1 & 1,2 & 1,0 & $-1,7$ & $-0,3$ & $-2,1$ & $-1,4$ \\
\hline Rumänien & $-1,9$ & 7,6 & 3,7 & 3,2 & $-7,5$ & 1,6 & $-2,1$ & 0,1 & $-2,8$ & 1,8 & $-4,1$ & $-0,8$ \\
\hline Slowakei & 3,6 & 4,4 & 0,9 & 2,3 & 2,7 & 3,7 & $-3,2$ & $-0,6$ & 5,7 & $-2,0$ & $-4,7$ & $-1,9$ \\
\hline Slowenien & 1,8 & 4,3 & 2,0 & 0,5 & 1,0 & 2,2 & $-0,1$ & $-1,7$ & 7,3 & $-1,8$ & $-1,7$ & $-2,0$ \\
\hline Tschechien & $-1,2$ & 3,7 & 1,6 & 2,0 & $-1,8$ & 2,5 & $-0,6$ & $-1,3$ & 1,7 & $-2,0$ & $-2,0$ & $-1,4$ \\
\hline Ungarn & $-1,4$ & $-2,3$ & 5,8 & 3,7 & $-5,4$ & $-7,1$ & 1,9 & $-1,8$ & $-1,2$ & $-8,0$ & 0,5 & $-0,5$ \\
\hline Gesamte EU 27 & 1,8 & 2,3 & 2,2 & 2,1 & 0,8 & 0,2 & $-0,9$ & $-0,5$ & 3,2 & $-2,3$ & $-2,3$ & $-0,8$ \\
\hline
\end{tabular}

*Nominallöhne $=$ Nominale Arbeitnehmerentgelte pro Arbeitnehmer, Veränderung in \% gegenüber dem Vorjahr

Reallöhne $=$ Nominale Arbeitnehmerentgelte pro Arbeitnehmer deflationiert um die Entwicklung des Harmonisierten Verbraucherpreisindex, Veränderungen in \% gegenüber dem Vorjahr.

Verteilungsbilanz $=$ Saldo des jährlichen Nominallohnzuwachses und des Verteilungsspielraums (vgl. Tabelle 2) in Prozentpunkten. Angaben für 2012: Prognose de Europäischen Kommission.

Quelle: AMECO Datenbank der Europäischen Kommission, Version: 11.5.2012, Berechnungen des WSI.

derzeit einer starke Tendenz zur Dezentralisierung und Verbetrieblichung aufweisen (Busch et al. 2012).

\section{Entwicklung der Effektivlöhne}

Bei der Analyse der Effektivlöhne wird im Folgenden erneut auf die AMECO-Datenbank der Europäischen Kommission zurückgegriffen, deren Ursprungsdaten aus den nationalen volkswirtschaftlichen Gesamtrechnungen stammen. ${ }^{\odot}$ 
2011 stiegen danach die effektiven Nominallöhne im EUDurchschnitt um 2,2 \%. Mit 2,3 \% im Jahr 2010 und prognostizierten 2,1 \% für 2012 zeigt die Nominallohnentwicklung damit in den letzten Jahren eine große Konstanz (Tabelle 3).

Hinter den EU-Durchschnittsdaten verbergen sich allerdings erhebliche nationale Unterschiede. Die höchsten Nominallohnzuwächse konnten 2011 einige osteuropäische Staaten verzeichnen, darunter als Spitzenreiter Bulgarien mit 7,3 \%, gefolgt von Ungarn mit 5,8 \% und Polen mit 5,1 \%. Außerhalb Osteuropas gab es die höchsten Nominallohnsteigerungen in Belgien, Deutschland und Österreich, wo die Zuwachsraten zwischen 3 und 3,3 \% variierten.

Insbesondere in den südeuropäischen Krisenstaaten stand die Lohnentwicklung dagegen stark unter Druck und hat - wenn überhaupt - nur zu geringen Nominallohnerhöhungen geführt. In drei Ländern war die Entwicklung des durchschnittlichen Nominallohniveaus sogar negativ, wobei der Rückgang mit minus 3,2 \% in Griechenland am stärksten ausgeprägt war, gefolgt von Irland mit minus 1,4 \% und Portugal mit minus 0,8 \%. Hinter dieser Entwicklung verbargen sich vor allem massive Lohnkürzungen im öffentlichen Dienst, die zunehmend auch auf die Privatwirtschaft ausstrahlen (Labour Research Department 2012; Vandekerckhove et al. 2012).

Für das Jahr 2012 erwartet die Europäische Kommission, dass sich die Politik der Lohnkürzungen in den genannten Staaten weiter fortsetzen wird. So wird für Griechenland ein Nominallohnrückgang von $8 \%$ prognostiziert. Eine negative Nominallohnentwicklung wird auch für Portugal (minus 3,1\%) sowie Irland und Zypern (jeweils minus $0,8 \%$ ) vorausgesagt, während in Spanien mit plus $0,1 \%$ das bestehende Nominallohnniveau faktisch eingefroren wird. Dagegen können die meisten osteuropäischen Länder mit überdurchschnittlich hohen Nominalzuwachsraten ihren Aufholprozess weiter fortsetzen. Relativ hohe Nominallohnsteigerungen von mehr als $3 \%$ werden auch für Belgien, Luxemburg, Finnland und Österreich erwartet. Deutschland befindet mit einer prognostizierten Zuwachsrate von 2,7\% im oberen Mittelfeld.

Vor dem Hintergrund vergleichsweise hoher Inflationsraten blieben im Jahr 2011 die Nominallöhne zumeist hinter den Preisen zurück, sodass die effektiven Reallöhne einen negativen Entwicklungstrend aufwiesen. Insgesamt mussten die Beschäftigten in 21 von 27 EU-Staaten Reallohnverluste hinnehmen. Im EU-Durchschnitt ging das Reallohnniveau um $0,9 \%$ zurück (Tabelle 3). Der europaweite Reallohnrückgang wäre dabei noch deutlich stärker ausgefallen, wenn nicht mit Deutschland und Frankreich die beiden größten europäischen Volkswirtschaften ein kleines Reallohnplus von jeweils 0,5\% verzeichnet hätten. Für 2012 erwartet die Europäische Kommission, dass sich die negative Reallohnentwicklung in 15 von 27 EU-Staaten und mit einem europaweiten Minus von 0,5\% weiter fortsetzen wird.

Angesichts sinkender Reallöhne ist es nicht weiter verwunderlich, dass auch die Verteilungsbilanz, die sich aus dem Saldo aus Nominallohnentwicklung und dem Verteilungsspielraum ergibt, 2011 mit Ausnahme von Ungarn in sämtlichen EU-Staaten negativ ausgefallen ist. Dies bedeutet, dass es nahezu keinem EU-Land gelungen ist, den lohnpolitischen Verteilungsspielraum aus Preis- und Produktivitätsentwicklung auszuschöpfen. Im EU-Durchschnitt lag die Verteilungsbilanz 2,3 Prozentpunkte unterhalb des Verteilungsspielraums. 2012 werden nach den Prognosen der Europäischen Kommmission immerhin wieder acht EUStaaten (darunter auch Deutschland) den Verteilungsspielraum (über)ausschöpfen. Für die gesamte EU bleibt die Verteilungsbilanz hingegen mit minus 0,8 Prozentpunkten negativ, sodass die Lohnquote weiter zurückgehen und sich die Umverteilung zugunsten der Kapitaleinkommen weiter fortsetzen wird.

\section{Ausblick: Anhaltende Reallohn- verluste in Europa}

Im Zuge der Weltwirtschaftskrise 2009ff. hat sich das lohnpolitische Entwicklungsmuster in Europa grundlegend verändert. Bis zum Ausbruch der Krise war die Lohnentwicklung durch mehr oder weniger hohe Reallohnzuwächse geprägt, die sich noch bis in das Krisenjahr 2009 fortsetzten. Im Zeitraum 2001 bis 2009 zeigten insbesondere die osteuropäischen Länder sehr hohe Reallohnsteigerungen und unterstrichen damit ihren ökonomischen Aufholprozess gegenüber den übrigen EU-Staaten (Abbildung 5). Außerhalb Osteuropas kam es in so unterschiedlichen Staaten wie Irland und Großbritannien, Griechenland sowie den nordeuropäischen Ländern Dänemark und Finnland zu relativ hohen Reallohnsteigerungen. Zu der Gruppe mit vergleichsweise niedrigen Reallohnzuwächsen gehörten dagegen vor allem die deutschen Nachbarstaaten Frankreich, Belgien, Luxemburg und Österreich, aber auch die südeuropäischen Staaten Italien, Portugal und Spanien. Eine absolute lohnpolitische Sonderrolle wurde schließlich von Deutschland eingenommen, das während der letzten Dekade als einziges Land in Europa erhebliche Reallohnrückgänge zu verzeichnen hatte (Schulten 2011b).

Seit 2010 zeigt die Lohnentwicklung in Europa ein vollkommen neues Muster, in dem Reallohnverluste zum dominierenden Trend werden. Unter Einbeziehung der Prognosedaten der Europäischen Kommission ist die Reallohnentwicklung im Zeitraum 2010 bis 2012 in 18 von 27 EU-Staaten rückläufig (Abbildung 6). Besonders extrem sind die Reallohnverluste mit mehr als $20 \%$ in Griechenland, gefolgt von Portugal mit mehr als $10 \%$ und Zypern mit mehr als $8 \%$. Hohe Reallohnrückgänge von mehr als $5 \%$ gab es darüber hinaus auch in Irland und Spanien sowie Lettland und Ungarn. Auf der anderen Seite bestehen mit Bulgarien und Polen lediglich zwei Staaten, in denen es im selben Zeitraum größere Reallohnzuwächse gab. Auch 
ABB. 5

\section{Entwicklung der Reallöhne in der EU 2001-2009}

Angaben in Prozent

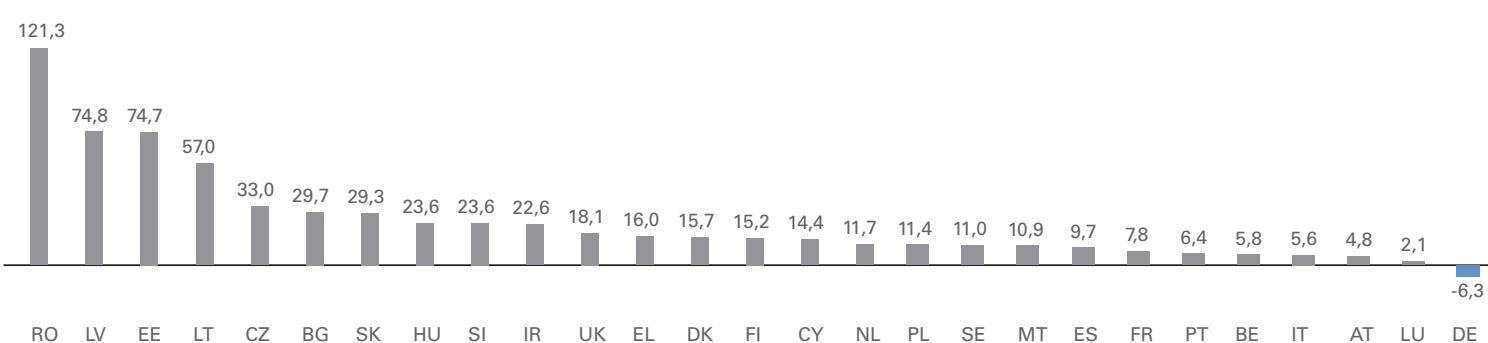

$\mathrm{AT}=$ Österreich, $\mathrm{BE}=$ Belgien, $\mathrm{CY}=$ Zypern, $\mathrm{CZ}=$ Tschechische Republik, DE = Deutschland, DK = Dänemark, EE = Estland, EL = Griechenland, ES = Spanien $\mathrm{FI}=$ Finnland, $\mathrm{FR}=$ Frankreich, $\mathrm{HU}=$ Ungarn, $\mathrm{IE}=$ Irland, IT = Italien, $\mathrm{LT}=$ Litauen, $\mathrm{LU}=$ Luxemburg, $\mathrm{LV}=\mathrm{Lettland}, \mathrm{MT}=\mathrm{Malta}, \mathrm{NL}=\mathrm{Niederlande}, \mathrm{PL}=\mathrm{Polen}$ $\mathrm{PT}=$ Portugal, $\mathrm{RO}=$ Rumänien, $\mathrm{SE}=$ Schweden, $\mathrm{SI}=$ Slowenien, $\mathrm{SK}=$ Slowakei, UK = Großbritannien

*deflationiert um den Harmonisierten Verbraucherpreisindex (HVPI).

Quelle: Ameco-Datenbank; Berechnungen des WSI.

\section{ABB. 6}

\section{Entwicklung der Reallöhne in der EU 2010-2012}

Angaben in Prozent

15,4

$\begin{array}{llllllll}5,4 & 2,2 & 1,8 & 1,4 & 0,9 & 0,6 & 0,4 & 0,3\end{array}$

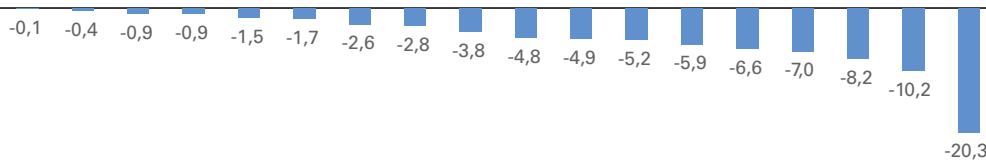
$\begin{array}{lllllllllllllllllllllllllllllll}B G & P L & S E & D E & F I & F R & C Z & \text { SI } & \text { AT } & \text { SK } & \text { RO } & \text { NL } & \text { BE } & \text { DK } & \text { LU } & \text { IT } & \text { UK } & \text { LT } & \text { EE } & \text { MT } & \text { LV } & \text { ES } & \text { IR } & \text { HU } & \text { CY } & \text { PT } & \text { EL }\end{array}$

$\mathrm{AT}=$ Österreich, $\mathrm{BE}=$ Belgien, $\mathrm{CY}=\mathrm{Zypern}, \mathrm{CZ}=\mathrm{Tschechische} \mathrm{Republik,} \mathrm{DE}=$ Deutschland, $\mathrm{DK}=$ Dänemark, EE = Estland, EL = Griechenland, $\mathrm{ES}=\mathrm{Spanien}$ $\mathrm{FI}=$ Finnland, $\mathrm{FR}=$ Frankreich, $\mathrm{HU}=$ Ungarn, IE = Irland, IT = Italien, $\mathrm{LT}=$ Litauen, $\mathrm{LU}=$ Luxemburg, $\mathrm{LV}=\mathrm{Lettland}, \mathrm{MT}=\mathrm{Malta}, \mathrm{NL}=\mathrm{Niederlande}, \mathrm{PL}=\mathrm{Polen}$ $\mathrm{PT}=$ Portugal, $\mathrm{RO}=$ Rumänien, $\mathrm{SE}=\mathrm{Schweden}, \mathrm{SI}=$ Slowenien, $\mathrm{SK}=$ Slowakei, UK = Großbritannien

*deflationiert um den Harmonisierten Verbraucherpreisindex (HVPI). Daten für 2012: Frühjahrsprognose der Europäischen Kommission.

Quelle: Ameco-Datenbank; Berechnungen des WSI.

Deutschland konnte erstmals seit Langem wieder eine positive Reallohnbilanz aufweisen, wobei hierdurch jedoch bislang nur ein kleiner Teil der Reallohnverluste der 2000er Jahre ausgeglichen werden konnte.

Als Reaktion auf die Krise kommt es in den meisten europäischen Ländern zu einer äußerst restriktiven Lohnpolitik, die in der Regel nur noch bescheidene Lohnzuwächse zulässt, oft zu einem Einfrieren gegebener Lohnniveaus führt und in einigen Staaten sogar mit offenen Lohnkürzungen einhergeht (Vandekerckhove et al. 2012). Der Staat spielt hierbei vielfach eine Vorreiterrolle, indem er vor allem über die Löhne im öffentlichen Sektor (Labour Research Department 2012) und die gesetzlichen Mindestlöhne (Schulten 2012) die allgemeine Lohnentwicklung direkt zu beeinflussen sucht. Hinzu kommt, dass angesichts des starken Anstiegs der Arbeitslosigkeit auch in der Privatwirtschaft die Bereitschaft zu lohnpolitischen Konzessionen immer größer und damit die Verhandlungsmacht der Gewerkschaften immer kleiner wird.

Von wachsender Bedeutung für die Lohnentwicklung in Europa ist schließlich auch die Rolle der EU, die regelrecht einen „neuen lohnpolitischen Interventionismus“ hervorgebracht hat, der lohnpolitische Auflagen mit der Androhung ökonomischer Sanktionen verbindet (Busch et al. 2012). Obwohl die EU laut EU-Vertrag (Artikel 153, Abs. 5) eigentlich im Bereich der Lohnpolitik gar keine Regelungskompetenzen hat, ist Letztere mit dem 2011 verabschiedeten Euro-Plus-Pakt offiziell zu einem zen- 
tralen europäischen Politikfeld erhoben worden (Europäischer Rat 2011). Der Euro-Plus-Pakt interpretiert die aktuelle Krise in Europa vor allem als eine Krise fehlender ökonomischer Wettbewerbsfähigkeit, für deren Wiederherstellung in erster Linie die Lohnpolitik verantwortlich gemacht wird (s.a. Europäische Kommission 2011). Dementsprechend werden mittlerweile von der EU im Rahmen des sogenannten „Europäischen Semesters" regelmäßig die Lohnentwicklungen in allen EU-Staaten überprüft und Empfehlungen für die nationale Lohnpolitik ausgesprochen. Letztere beziehen sich nicht nur auf die Höhe einer „angemessenen“ Entwicklung der Löhne und Lohnstückkosten, sondern auch auf die Reform der Tarifvertragssysteme, wobei in der Regel eine mehr oder weniger weitreichende Dezentralisierung empfohlen wird. Den stärksten Einfluss auf die nationale Lohnpolitik hat die EU derzeit in denjenigen Ländern, wo sie im Rahmen der „Troika“ zusammen mit der Europäischen Zentralbank und dem Internationalen Währungsfonds bestimmte lohnpolitische Maßnahmen wie Kürzung von Löhnen und Dezentralisierung der Tarifvertragssysteme als Vorbedingungen für den Erhalt von finanziellen Mitteln aus dem Europäischen Rettungsfonds festlegen kann (Busch et al. 2012). Dies hat mit dazu beigetragen, dass in Ländern wie Griechenland, Portugal oder Irland der Einbruch bei den Reallöhnen seit einigen Jahren am stärksten ist.

Die jüngste Reallohnentwicklung in Europa deutet bereits an, wie die Unterordnung der Lohnpolitik unter die Wettbewerbspolitik einen lohnpolitischen Absenkungswettlauf in Gang setzt. Nach dem Vorbild Deutschlands sollen Lohnmoderation und Reallohnverluste zu neuer Wettbewerbsfähigkeit führen und dadurch einen Weg aus der Krise ebnen. Das extrem einseitig auf Export ausgerichtete deutsche Entwicklungsmodel der 2000er Jahre ist jedoch in einem Europa, in dem sich immer noch mehr als zwei Drittel seiner Handels- und Zahlungsströme innerhalb der EU-Grenzen bewegen, nicht verallgemeinerbar. Der vermeintliche Gewinn an preislicher Wettbewerbsfähigkeit durch eine restriktive Lohnpolitik wird im Gegenteil in der EU insgesamt durch einen entsprechenden Verlust an gesamtwirtschaftlicher Nachfrage mehr als überkompensiert (Stockhammer/Onaran 2012). Schon heute müssen die export-orientierten Volkswirtschaften Westund Nordeuropas feststellen, dass die ökonomische Stagnation vor allem im Süden auch für sie nicht ohne Rückwirkungen bleibt.

Die bestehenden ökonomischen Ungleichgewichte innerhalb Europas können nicht allein durch Anpassungen in den Krisen- und Defizitländern und schon gar nicht allein durch deren Lohnpolitik abgebaut werden. Die Lohnentwicklung in diesen Ländern müsste vielmehr dazu beitragen, den anhaltenden Reallohnverlust zu stoppen, damit die gesamtwirtschaftliche Nachfrage nicht weiter einbricht. Die west- und nordeuropäischen Überschussländer stehen hingegen vor der Aufgabe, auch mithilfe einer in den nächsten Jahren deutlich expansiveren Lohnentwicklung die wirtschaftliche Dynamik in ganz Europa zu fördern. Die jüngsten Lohndaten aus Deutschland deuten hierbei immerhin auf einen gewissen Richtungswechsel. Schließlich hätte auch die EU eine neue lohnpolitische Rolle zu übernehmen, die jedoch nicht mehr in der Propagierung eines europaweiten Lohnsenkungswettbewerbs als vielmehr in einer solidarischen und wachstumsorientierten Koordinierung der Lohnpolitik liegen müsste.

\section{LITERATUR}

Aumayr, C./Cabrita, J./Demetriades, S. (2011): Pay developments - 2010, Euro found, Dublin

Busch, K./Hermann, C./Hinrichs, K./Schulten, T. (2012): Austeritätspolitik in der Eurokrise und das Europäische Sozialmodell, Studie im Auftrag der FriedrichEbert-Stiftung, Berlin (im Erscheinen)

Deutsche Bank Research (2011): Deutschland: Nicht länger die Insel der Glückseligen, Reihe Aktuelle Themen (523), 22. August

Europäische Kommission (2011): Assessing the Links between Wage setting, Competitiveness and Imbalance, Note for the Economic Policy Committee, Brüssel, http://gesd.free.fr/wagecomp11.pdf

Europäische Kommission (2012): European Economic Forecast - Spring 2012 European Economy (1), May, Brussels, http://ec.europa.eu/economy_finance/ publications/european_economy/2012/pdf/ee-2012-1_en.pdf

Europäischer Rat (2011): Der Euro-Plus-Pakt. Stärkere Koordinierung der Wirtschaftspolitik im Hinblick auf Wettbewerbsfähigkeit und Konvergenz, Schlussfolgerungen des Europäischen Rats vom 24./25. März, Brüssel

Eurostat (2012): Mai 2012: Arbeitslosenquote des Euroraums bei 11,1 \%. Quote der EU27 bei 10,3\%, Pressemitteilung Nr. 101 vom 2. Juli, Luxemburg Herr, H./Horn, G. A. (2012): Lohnpolitik heute, IMK Policy Brief, Mai, Düsseldorf Institut für Makroökonomie und Konjunkturforschung (IMK)/L'Observatoire Français des Conjonctures Économiques (OFCE)/Österreichisches Institut für Wirtschaftsforschung (WIFO) (2012): Fiskalpakt belastete Euroraum. Gemeinsame Diagnose des Makro-Konsortiums, IMK Report (71), März, Düsseldorf Keune, M. (2011): Decentralising Wage Setting in Times of Crisis? The Regulation and Use of wage-related Derogation Clauses in seven European Countries, in: European Labour Law Journal 2 (1), S. 86-96

Labour Research Department (2012): The Wrong Target one year on: Pay Cuts in the Public Sector in the European Union, Studie im Auftrag der Europäischen Gewerkschaftsverbandes für den Öffentlichen Dienst (EGÖD), http://www. epsu.org/a/8828

Schubert, A. (2012): Experimental data as part of the ECB's statistical production and dissemination policy, Papier präsentiert auf der European Conference on Quality in Official Statistics, 29. Mai - 1. Juni in Athen, http://www. q2012.gr/articlefiles/sessions/26.2-Experimental-statisticsECB-Aurel-Schubert. pdf

Schulten, T. (2010): Das deutsche Tarifvertragssystem im europäischen Vergleich, in: Bispinck, R./Schulten, T. (Hrsg.): Zukunft derTarifautonomie, Hamburg, S. 193-204

Schulten, T. (2011a): Europäischer Tarifbericht 2010/2011, in: WSI-Mitteilungen 64 (7), S. 355-362, http://www.boeckler.de/wsimit_2011_07_schulten.pdf Schulten, T. (2011b): Deutschlands lohnpolitische Sonderrolle in Europa, in Bispinck, R. (Hrsg.): Zwischen Beschäftigungswunder und Lohndumping? Tarifpolitik in und nach der Krise, Hamburg, S. 47-58

Schulten, T. (2012): WSI-Mindestlohnbericht 2012 - Schwache Mindestlohnentwicklung unter staatlicher Austeritätspolitik, in: WSI-Mitteilungen 65 (2),

S. 124-130, http://www.boeckler.de/wsimit_2012_02_schulten.pdf

Stockhammer, E./Onaran, Ö. (2012): Rethinking wage policy in the face of the Euro crisis: Implications of the wage-led demand regime, in: International Review of Applied Economics 26 (2), S. 191-203

Vandekerckhove, S./van Peteghem, J./van Gyes, G. (2012): Wages and Working Conditions in the Crisis, Eurofound, Dublin

\section{AUTOR}

THORSTEN SCHULTEN, Dr., Wissenschaftler im Wirtschafts- und Sozialwissen schaftlichen Institut (WSI) der Hans-Böckler-Stiftung. Arbeitsschwerpunkte: Arbeits- und Tarifpolitik in Europa.

thorsten-schulten@boeckler.de 